\title{
Does Test Dose of Central Stimulant Influence Continuous Performance Test (CPT) and Activity in Boys with Attention Deficit Hyperactivity Disorder?
}

\author{
${ }^{1}$ Unit for Children and Adolescents, Regional Hospital Viborg, Denmark \\ ${ }^{2}$ Department of Child and Adolescent Psychiatry, Kolding, Denmark \\ ${ }^{3}$ Department of Child and Adolescent Psychiatry, Odense, Denmark
}

Merete Eybye Dam ${ }^{1}$, Katrine Kaastrup Kolmos ${ }^{2}$ and Niels Bilenberg ${ }^{3}$

*Corresponding author: Merete Dam, Unit for Children and Adolescents, Regional Hospital Viborg, Denmark, Tel: +45 27268201; E-mail: merete.dam@hotmail.com

Received date: Sep 1, 2016; Accepted date: Sep 2, 2016; Published date: Sep 8, 2016

Copyright: (C) 2016 Dam ME et al. This is an open-access article distributed under the terms of the Creative Commons Attribution License, which permits unrestricted use, distribution, and reproduction in any medium, provided the original author and source are credited.

Citation: Dam ME, Kolmos KK, Bilenberg N. Does Test Dose of Central Stimulant Influence Continuous Performance Test (CPT) and Activity in Boys with Attention Deficit Hyperactivity Disorder? Clin Psychiatry. 2016, 2:3.

\section{Abstract}

Background: Monitoring the effect of medication by using an objective measure would bring new perspectives to the work with patients suffering from Attention Deficit Hyperactivity Disorder (ADHD).

Objective: In order to objectify and monitor treatment efficiency, a Continuous Performance Test (the QB Test) was used and evaluated in daily clinical practice.

Method: A total of 12 boys aged 8-12 fulfilling diagnostic criteria for ADHD were tested using the $\mathrm{QB}$ Test before and after intake of methylphenidate.

Findings: One hour after intake of test dose of medicine, there were significant improvements on numbers of omissions $(p=0.014)$, reaction time $(p=0.038)$, correct responses $(p=0.031)$ and activity $(p=0.045)$. Nonsignificant improvements in reaction time variability $(p=0.190)$ and correct non-responses $(p=0.764)$ were found. Number of guesses and errors of commission were unchanged after medication.

Conclusions: The QB Test can be used to track effects of medication, showing greatest improvement in the most inattentive and hyperactive/impulsive children.

Keywords: ADHD, CPT, QB test, Methylphenidate

\section{Introduction}

Attention Deficit Hyperactivity Disorder (ADHD) (DSM-IV and DSM-5) or Hyperkinetic Disorder (ICD-10) is a neurodevelopmental psychiatric disorder that causes attention deficits, hyperactivity, and/or impulsiveness [1,2].

To fulfill diagnostic criteria, the symptom debut must be before age 7 and persist for more than 6 months. The symptoms can be present to varying degrees but must impact the patient's life in at least two different settings, e.g. school and home [1-3].

ADHD is the most commonly studied and diagnosed psychiatric disorder in children and adolescents. It occurs in 3-5\% of Danish children, and in $15-20 \%$ of the children referred to Child and Adolescent Psychiatry. ADHD is 4 to 5 times more common in boys than girls, and in half of the patients it persists into adulthood [4,5].

According to NICE Guidelines [3], the diagnosis should only be made by a psychiatrist, pediatrician or other appropriately qualified healthcare professional. The diagnostic process requires a full clinical and psychosocial assessment, and observer reports from different life settings. Information from parents about developmental and psychiatric history is important, as well as information from school or kindergarten teachers.

Rating scales such as the Conner's rating scale, ADHD-rating scale (-RS), the Child Behaviour Checklist ( $C B C L)$ and the Strengths and Difficulties Questionnaire (SDQ) are valuable adjunct tools [3].

First-line treatment for school-aged children with ADHD and moderate impairment is group-based parent-training education programmes, behavioural and social therapy. However, in some cases, this will be insufficient, and the child will need drug treatment. For children with severe ADHD and severe impairment, drug treatment is first-line treatment [3]. The recommended first-line drug is methylphenidate, which is the most frequently used drug for ADHD in Denmark $[6,7]$.

In an attempt to support clinical descriptive diagnostics and systematic monitoring of treatment, cognitive tests in different set-ups have been investigated [8-12]. Continuous Performance Test (CPT) is an overall general category of tests that can be used in many contexts, including ADHD [13].

A review from 2004 gives a summary of the use of different cognitive tests (Continuous Performance Test, the Gordon 
Diagnostic System, the Children's Checking Task, Delay of Gratification Tasks, the Choice-Delay Task, and the Stop Signal Task (SST) [14].

CPT is able to discriminate ADHD from a control group, but has a large number of false positive and false negative results. This indicates that CPT is not sufficient to reliably distinguish between ADHD and non-ADHD subjects or to differentiate ADHD from other mental disorders [14].

In a number of studies, methylphenidate is shown to improve symptoms measured on CPT to the extent that children with ADHD treated with methylphenidate can perform nearly as well as a control group [9,12,14-18].

There is ongoing research into CPT and its ability to support diagnostics, monitoring of symptom severity and evaluation of medication. In an attempt to assess all three core symptoms of $\mathrm{ADHD}$, the tracking of movement during tests has come into focus $[4,6,8,10,12,19]$.

Others have investigated the use of CPT in a virtual setting or as an online test that can be made in the child's home or at school, to create a naturalistic setting $[11,17]$.

The Child and Adolescent Psychiatry Unit in Odense uses a variant of CPT called the QB Test (Quantitative Behavioural Test). This is a CPT combined with an activity test, which makes it possible to establish a picture of the complexity of the disease $[10,19]$.

The purpose of this study was to investigate the objective effect on ADHD core symptoms after intake of a test-dose of methylphenidate. Participants who already fulfill diagnostic criteria of ADHD were grouped on the basis of parent rating on ADHD-RS. Change in attention function and activity was measured by QB Test.

\section{Method}

Data used in the study was generated from the QB testing of 13 boys in the Child and Adolescent Psychiatry Outpatient Clinic in Odense and the ADHD Rating Scale (ADHD-RS) completed by their parents.

The cases included were boys born in 1999-2003 and diagnosed with ADHD, all in daily treatment with methylphenidate. The treatment duration varied from months to years.

Boys with a diagnose of intellectual disability and other psychiatric comorbidity were excluded from the study. So were boys treated with medication other than methylphenidate because, due to the significantly longer half-life of other active substances, they would not be able to comply with the medication fasting that was required in this study.

Prior to this study no new IQ measure were made.

There were 30 boys who met the criteria for participation; 13 of them (and their parents) gave consent to participation.

On the day of the trial, the participants attended with a parent or caregiver without having taken their usual morning medication. They all had a medication washout from the day before.

Prior to the execution of the actual test, a presentation video was displayed, and the children received some initial training in the program until they were able to demonstrate that they understood the three aspects of the test; to sit still, correct and fast pressing and correct non-pressing.

The QB Test was conducted twice $(2 \times 15 \mathrm{~min})$; once without medication and the other an hour after intake of their usual morning medication.

During the test, the participants and one of two evaluators sat in a quiet room in front of a computer, wearing a sensor to detect activity. No interaction between test-person and evaluator took place during the test. Evaluators were authors Merete Dam and Katrine Kolmos.

All participants had already tried the QB Test when diagnosed, or in connection with drug monitoring. We assume this will not have affected the outcome since it is not possibly to improve results by repetition. Test retest studies of QB Test show high reliability [20].

\section{QB Test}

The QB Test is a computer program that can test a person's attention, activity level and impulsivity and thus generate an objective measure of ADHD core symptom severity.

The task of the program is to press a button at a specific stimulus, and refrain at another. In the course of 15 minutes, the person is shown 225 targets and 225 non-targets [19].

After completing the test, the QB Test program outlines a summary of the test person's score and compares the data to an age-matched control group.

Inattention:

- Reaction time (RT) is the average of elapsed time from target presentation to response.

- Variability in reaction time (RT-var) is the fluctuation of reaction time during the test.

- Errors of omission are the number of non-responses to a target.

- Correct responses are the number of responses to a target.

- Correct non-responses are when refraining from response to a non-target [10].

Impulsiveness and hyperactivity:

- Guesses are measured as the number of hits before a test person is able to identify a target or non-target.

- Errors of commission are the number of responses to a non-target $[6,12,18]$.

- Activity is measured as the distance a test person moves his head from baseline. This distance is registered by an infrared camera and is shown as movement around a centre of a coordinate $\left(\mathrm{cm}^{2}\right)[10,19]$. 


\section{ADHD - RS}

ADHD-RS is a questionnaire in which parents rate the presence of 18 symptoms on a Likert scale from "never" (0) to "very often" (3). ADHD-RS gives information on activity, inattentiveness and impulsivity [18]. The rating scale is primarily used to monitor treatment efficiency, but also plays an important role in the diagnostic process $[5,7,9]$.

\section{Methylphenidate}

Methylphenidate is a central nervous system stimulant used in treatment of ADHD. It blocks the reabsorption of dopamine and increases the activity in the frontal lobes and basal ganglia. Initially the dose is titrated from $5 \mathrm{mg}$ twice a day to the optimal level of up to $1.2-1.5 \mathrm{mg} / \mathrm{kg} /$ day that achieves therapeutic benefits and has minimal side effects.

Duration of effect varies from 3-12 hours due to difference in release. Half-life is around 2-3 hours. Annual review of drug dose, benefits, side effects, growth and overall monitoring is recommended [3] (Table 1).

Table 1 Characteristics of the test sample $(\mathrm{N}=13)$.

\begin{tabular}{|l|l|l|}
\hline & Min-max & Mean (SD) \\
\hline ADHD-RS inattention & $8-19$ & 14.2 \\
\hline ADHD-RD hyperactivity/impulsivity & $4-19$ & 13.6 \\
\hline Age & $8-12$ & 10.5 \\
\hline Daily dose of Methylphenidate $(\mathrm{mg})$ & $20-64$ & 37.6 \\
\hline
\end{tabular}

The participants in this study received varying doses (20-64 $\mathrm{mg}$ ), but all had had monitoring of their drug treatment within the last year.

\section{Statistical tests and ethics}

All analyses were conducted using SPSS ver. 19. Data from $\mathrm{QB}$ testing prior to and after methylphenidate intake were compared using the paired t-test. Data was normally distributed. Statistical significance is defined as $p<0.05$.

The study is designed as a pilot project with the approval of the research ethics committee in the Region of Southern Denmark (Project ID S - 20110093).

\section{Results}

13 boys completed the QB Test twice and had their symptoms rated on the ADHD-RS.

One boy was excluded because of oppositional behaviour that led to obvious mistakes made on purpose. This was considered not to reflect the true severity of his symptoms, and therefore the data from this participant were not included in any analysis (Table 2).

ADHD-RS is used to find those who are likely or most likely to be inattentive and/or hyperactive/impulsive. According to the Danish version for interpretation of ADHD-RS, the cut-off is a score of 9 or more on a given subscale. Scores between 9 and 13 constitute a grey zone that requires more diagnostics.

A person scoring above 13 is likely to have an attention and/or hyperactivity/impulsivity deficit (Table 3).

Table 2 Paired sample T-test.

\begin{tabular}{|c|c|c|c|c|c|c|c|}
\hline \multirow[t]{2}{*}{ Attention measures $\mathrm{N}=12$} & \multicolumn{3}{|c|}{ Before Methylphenidate } & \multicolumn{3}{|c|}{ After Methylphenidate } & \multirow{2}{*}{$\begin{array}{l}\text { T-test } \\
\text { Sig. 2-tailed }\end{array}$} \\
\hline & Mean & Min & Max & Mean & Min & Max & \\
\hline RT & 467.25 & 323 & 694 & 440.08 & 305 & 631 & $0.038^{*}$ \\
\hline RT-var & 155.08 & 112 & 381 & 144.17 & 92 & 421 & 0.19 \\
\hline Omission & 29.92 & 2 & 112 & 10.58 & 2 & 41 & $0.014^{*}$ \\
\hline Correct response & 186.58 & 84 & 223 & 204 & 163 & 223 & $0.031^{*}$ \\
\hline Correct non-response & 177.33 & 86 & 221 & 176.25 & 65 & 220 & 0.764 \\
\hline
\end{tabular}

Table 3 Paired sample T-test.

\begin{tabular}{|c|c|c|c|c|c|c|c|}
\hline \multirow[t]{2}{*}{ Hyperactivity/impulsiveness $\mathrm{N}=10$} & \multicolumn{3}{|c|}{ Before Methylphenidate } & \multicolumn{3}{|c|}{ After Methylphenidate } & \multirow{2}{*}{$\begin{array}{l}\text { T-test } \\
\text { Sig. 2-tailed }\end{array}$} \\
\hline & Mean & Min & Max & Mean & Min & Max & \\
\hline Activity & 113.6 & 23 & 234 & 73.3 & 28 & 162 & $0.045^{*}$ \\
\hline Guesses & 21.9 & 0 & 71 & 25.3 & 0 & 70 & 0.475 \\
\hline
\end{tabular}




\begin{tabular}{|l|l|l|l|l|l|l|l|}
\hline Commission errors & 42.8 & 3 & 123 & 44.2 & 5 & 124 & 0.684 \\
\hline${ }^{*}=\mathrm{P}<0.05$ & \\
\hline
\end{tabular}

12 participants had an inattention score of 9 or more, and 10 had a hyperactivity/impulsivity score of 9 or more and were therefore included in the statistical analyses.

\section{Discussion}

The findings of this study show significant improvements on numbers of omission $(p=0.014)$, reaction time $(p=0.038)$ and correct responses $(p=0.031)$. This shows that the boys are less inattentive after intake of methylphenidate. It also indicates that the participants have increased sustained attention after medication.

No significant improvements in reaction time variability $(p=0.190)$ or correct non-responses $(p=0.764)$ were found. This shows that the participants' fluctuation in reaction time had not improved significantly on the QB Test after intake of methylphenidate. The number of correct non-responses remained the same before and after intake of methylphenidate.

In this study, we found improvement in hyperactivity after intake of methylphenidate. The activity of the participants decreased from 113 to $\left.73 \mathrm{~cm}^{2} \mathrm{p}=0.045\right)$. The findings showed no improvement in the parameters reflecting impulsivity. The number of guesses and errors of commission were unchanged after medication.

A meta-analytic review from 1996 examined how results differed on the CPT in a group of children with ADHD with or without treatment with methylphenidate. The review concluded that omission and errors of commission were significantly reduced by $39 \%$ and $29 \%$ respectively in the group treated with methylphenidate compared to the group that received placebo [21]

The cases in this study had significant improvement of omission but not of errors of commission measured on the QB Test. This difference in findings may be due to the difference in study design, or the possibility that the QB Test is insufficient to assess the effect of methylphenidate on impulsivity.

Zalsmann and Solanto examined whether there was a difference in medication effect on the different subtypes of ADHD and found that there was a significant improvement after treatment with methylphenidate in all three subgroups, but no significant difference between them $[22,23]$.

In this study we found that both the most inattentive and hyperactive/impulsive improved significantly on some outcomes. Similar to Zalsmann and Solanto, this study did not find the improvements of the two groups to be largely different.

Heiser et al. discussed the correlation between the dose of methylphenidate and various CPT parameters. They found that "micro-events" as a sign of hyperactivity and RT variability as a sign of inattentiveness were significantly correlated to dose [4].

In a study from 2012, Gunther et al. found that a long-acting methylphenidate was as effective as two doses of immediaterelease methylphenidate in reducing inattention and hyperactivity during the course of the day. Impulsivity was reduced on immediate-release but not on long-acting methylphenidate [19].

Like Gunther et al., our study showed significant improvements on some inattention parameters and hyperactivity, but not impulsivity. Fluctuation of reaction time was unchanged before and after methylphenidate.

It is likely that RT variability would have been significantly improved measured on QB Test if the participants' dose of methylphenidate had been adjusted just ahead of testing or if the participants had been tested at another time during the day.

Another explanation for the lack of significance in improvement of RT variability could be that the test is not suitable for children, simply because they get bored and unfocused, which makes it impossible to track the effect of methylphenidate.

In recent years, there has been increased focus on developing tools for objective evaluation of hyperactivity. A Danish study by Tabori-Kraft, Sørensen et al. used an infrared camera comparable to the one used in the QB Test. They examined whether the OPTAx was able to show effect of methylphenidate. They found significant improvements of hyperactivity and impulsivity on all parameters. On inattentiveness, they found significant improvements of RT variability, correct pressing and correct non-pressing, but no improvements of RT or omission [6].

We expected results from the QB Test to point in the same direction because of the similarity of design and aim. The QB Test showed the same improvement of hyperactivity, but no improvements on guesses or errors of commission as an expression of repression of impulsivity.

Inattentiveness (RT, correct pressing and omissions) was significantly improved after methylphenidate on the QB Test.

The study of OPTAx and the QB Test share only some results. This may be explained by the larger sample size in the OPTAx study, and the fact that all of their participants were evaluated shortly before the trial regarding both diagnosis and medication dose.

Some studies focus on RT and RT variability [24]. When comparing children with ADHD with age-adjusted controls, it is found that they have longer RT and greater RT variability as a symptom of decreased overall attention but also a lack of sustained attention [9,11,23-25]. 
QB Test finds a significant improvement of reaction time, indicating an increased sustained attention ( $p=0.038)$.

The variability in reaction time has provided the basis for a study which indicates that a great RT variability is able to predict a bad response to methylphenidate [24,25].

In this study, no link between great RT variability and a lower improvement on the parameters of the three core symptoms was found when using the QB Test. There is a possibility that the sample size of this study is too small to show a connection between insufficient effect of medication and a certain parameter of the QB Test.

The variant of CPT used in the Region of Southern Denmark is the QB Test. In this test, the number of correct and noncorrect pressings is not taken into consideration. It is possible that these two outcomes, in addition to the ones already used, could shed some light on the effect of medication. In other studies, a concept known as accuracy is also being used as a measure of attention $[6,10]$. Accuracy is measured as the percentage of correct responses to both targets and nontargets, but this is not a part of the final analyses of the $Q B$ Test.

The results of this study must be interpreted in the context of the following limitations. First of all, the size of the sample is small. This may mean that we do not have the statistical power to obtain significant results on the QB Test, even if the participants do in fact have effect of their medication. In a small sample, it only takes a few participants with outlying results to blur effect or lack of effect of medication on the core symptoms of ADHD. In a study with 8 outcomes, the risk of coincidental results due to multiple comparisons needs to be considered. With a significance level of 0.05 , it is not unlikely that 1 of our 8 outcomes is false.

In addition, it would have made the setting more accurate if the participants had had a medication adjustment shortly before the QB testing.

Furthermore, there was no investigation of the motivation of the participants. It was obvious that some were very bored during the QB Test, and this may have caused the results to be obscured by inattentiveness caused by boredom rather than lack of medication effect.

It is difficult to imagine an entertaining computer-based program that does not allow the participants to improve by repetition, but despite several attempts requires them still to be willing to make an effort.

The purpose of the study was to investigate the effect of methylphenidate on QB Test in a group of boys that have ADHD of varying severity (rated by parents on ADHD-RS) of the 3 core symptoms. The results of this study show that it is possible to see improvements from methylphenidate on certain parameters of the QB Test. Overall, there was an improvement in attentiveness in those most inattentive, and in activity in those most hyperactive. It was not possible to find significant improvement in impulsivity on the QB Test.
Thus the study also demonstrates the challenge inherent in this type of test because the participants had trouble staying focused on the test for the entire time. This might be explained by their condition, but it might also be due to the configuration of the test.

In a clinical view, we do not find that the QB Test can stand alone in the diagnostic process, evaluation of symptoms or monitoring effects of medication. The QB Test can, however, help clarify the existence and/or severity of the core symptoms of ADHD.

It is important also to look at the data without using the $Q B$ Test program's interpretation. In this way, it is possible to take more aspects of attention into consideration. By using the number of correct responses and correct non-responses in relation to the total number of responses, it is possible to get a measure of accuracy.

The illustrative images that the QB Test generates might also be useful as a tool when teaching parents and/or children.

\section{References}

1. International Classification of Diseases (ICD-10).

2. Diagnostic and Statistical Manual of Mental Disorders (DSM IV).

3. https://www.nice.org.uk/guidance/conditions-and-diseases/ mental-health-and-behavioural-conditions/attention-deficitdisorder

4. Heiser P, Frey J (2004) Objective measurement of hyperactivity, impulsivity, and inattention in children with hyperkinetic disorders before and after treatment with methylphenidate. Eur Child Adolesc Psychiatry 13: 100-104.

5. http://www.bupnet.dk/media/ ADHD_faglige_retningslinier_revideret_26.06.12.pdf

6. Tabori-Kraft J, Sørensen MJ (2007) Is OPTAx useful for monitoring the effect of stimulants on hyperactivity and inattention? A brief report. Eur Child Adolesc Psychiatry 16: 347-351.

7. https://sundhedsstyrelsen.dk/da/nyheder/2015/ /media/ 91742BB2537F480E9481E415144C3687.ashx

8. O'Mahony N, Florentino-Liano B, Carballo JJ, Baca-Garcia E, Rodriguez AA (2014) Objective diagnosis of ADHD using IMUs. Med Eng Phys 36: 922-926.

9. Park S, Kim BN, Cho SC, Kim JW, Shin MS, et al. (2013) Baseline severity of parent-perceived inattentiveness is predictive of the difference between subjective and objective methylphenidate responses in children with attention-deficit/hyperactivity disorder. J Child Adolesc Psychopharmacol 23: 410-414.

10. Reh V, Schmidt M, Rief W, Christiansen H (2014) Preliminary evidence for altered motion tracking-based hyperactivity in ADHD siblings. Behav Brain Funct 13: 7-10.

11. Raz S, Bar-Haim Y, Sadeh A, Dan O (2014) Reliability and validity of the online continuous performance test among young adults. Assessment 21: 108-118.

12. Wehmeier PM, Schacht A, Wolff C, Otto WR, Dittmann RW, et al. (2011) Neuropsychological outcomes across the day in children with attention-deficit/hyperactivity disorder treated with atomoxetine: results from a placebo-controlled study using a 
computer-based continuous performance test combined with an infra-red motion-tracking device. J Child Adolesc Psychopharmacol 21: 433-444.

13. Egeland J, Kovalik-Gran I (2010) Validity of the factor structure of Conners' CPT. J Atten Disord 13: 347-357.

14. Nichols SL, Waschbusch DA (2004) A review of the validity of laboratory cognitive tasks used to assess symptoms of ADHD. Child Psychiatry Hum Dev 34: 297-315.

15. Wehmeier PM, Schacht A, Ulberstad F, Lehmann M, SchneiderFresenius C, et al. (2012) Does atomoxetine improve executive function, inhibitory control, and hyperactivity? Results from a placebo-controlled trial using quantitative measurement technology. J Clin Psychopharmacol 32: 653-660.

16. Meftagh SD, Najimi A, Mohammadi N, Ghanizadeh A, Rahimi C, et al. (2014) The most effective intervention for attention deficit-hyperactivity disorder: using continuous performance test. Psychiatr Danub 26: 165-171.

17. Pollak Y, Shomaly HB, Weiss PL, Rizzo AA, Gross-Tsur V (2010) Methylphenidate effect in children with ADHD can be measured by an ecologically valid continuous performance test embedded in virtual reality. CNS Spectr 15: 125-130.

18. Bart O, Daniel L, Dan O, Bar-Haim Y (2013) Influence of methylphenidate on motor performance and attention in children with developmental coordination disorder and attention deficit hyperactive disorder. Res Dev Disabil 34: 1922-1927.
19. Gunther T, Kahraman-Lanzerath B, Knospe EL, HerpertzDahlmann B, Konrad K (2012) Modulation of attention-deficit/ hyperactivity disorder symptoms by short- and long-acting methylphenidate over the course of a day. J Child Adolesc Psychopharmacol 22: 131-138.

20. Ulberstad F (2012) Test retest reliability for the Quantifed Behavioral Test (QbTest) in children.

21. Losier BJ, McGrath PJ (1996) Error patterns on the continuous performance test in non-medicated and medicated samples of children with and without ADHD: a meta-analytic review. J Child Psychol Psychiatry 37: 971-987.

22. Solanto M, Newcorn J, Vail L, Gilbert S, Ivanov I, et al. (2009) Stimulant drug response in the predominantly inattentive and combined subtypes of attention-deficit/hyperactivity disorder. J Child Adolesc Psychopharmacol 19: 663-671.

23. Zalsman G, Pumeranz O (2003) Attention patterns in children with attention deficit disorder with or without hyperactivity. Scientific World J 13: 1093-1107.

24. Epstein JN, Conners CK, Hervey AS, Tonev ST, Arnold LE, et al. (2006) Assessing medication effects in the MTA study using neuropsychological outcomes. J Child Psychol Psychiatry 47: 446-456.

25. Lee SH, Song DH, Kim BN, Joung YS, Ha EH, et al. (2009) Variability of response time as a predictor of methylphenidate treatment response in Korean children with attention deficit hyperactivity disorder. Yonsei Med J 50: 650-655. 